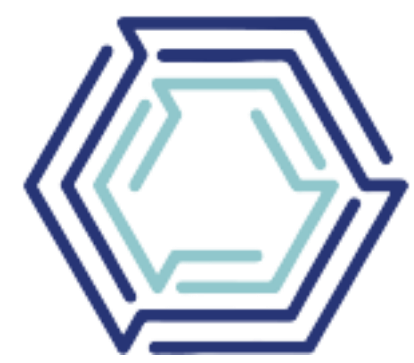

$\checkmark$ CONGRESSO INTERNACIONAL DAS LICENCIATURAS COINTER - PDVL 2018

\title{
SIMULADORES PARA SMARTPHONES: UMA PROPOSTA PARA O ENSINO DO ELETROMAGNETISMO E CIRCUITOS ELÉTRICOS
}

\section{SIMULATORS FOR SMARTPHONES: A PROPOSAL FOR THE TEACHING OF ELECTROMAGNETISM AND ELECTRICAL CIRCUITS}

\author{
Apresentação: Comunicação Oral \\ Marcos Antônio Vieira da Silva ${ }^{1}$; Antônio Edenilton Leite da Silva² ${ }^{2}$ Jailson da Silva \\ Soares $^{3}$; Isaiane Rocha Bezerra ${ }^{4}$; Haroldo Reis Alves de Macêdo ${ }^{5}$
}

DOI: https://doi.org/10.31692/2358-9728.VCOINTERPDVL.2018.00146

\begin{abstract}
Resumo
Com o constante advento de evoluções das Tecnologias Digitais de Informação e Comunicação, há uma necessidade da comunidade escolar em absorver e se utilizar desses avanços a fim de promover uma renovação didática e pedagógica, além de seguir as orientações das Leis Educacionais, como a Lei de Diretrizes e Bases da Educação Nacional (LDB) que cita a promoção do conhecimento tecnológico e científico. No ensino de Física, a experimentação é de fundamental importância pois explicita os conceitos e teorias através da prática, e dinamiza as aulas fornecendo a visão científica da área, superando as metodologias tradicionais. Este artigo tem por objetivo propor uma alternativa didática para o ensino de Física através de simulações de experimentos com smartphones. O aplicativo Physics Lab, alvo do estudo, foi caracterizado dentro do seu potencial uso como instrumento didático por fornecer simulações de experimentos físicos de maneira intuitiva e realista. Através da plataforma Android, obteve-se uma visão pedagógica de seu uso como auxiliar no ensino do Eletromagnetismo e dos Circuitos Elétricos, destacando pontos fortes e fracos dentro de categorias que podem nortear seu uso em sala de aula. A partir deste estudo, verificou-se que as simulações fornecidas pelo aplicativo são valiosas e podem complementar a abordagem teórica superando uma presumível falta de estrutura física de laboratórios específicos. O preparo e orientação do professor é de fundamental importância para o sucesso da proposta, pois atua como ponte entre os alunos e o conhecimento teórico-prático. Além disso, exemplifica-se possíveis métodos de uso do aplicativo oferecendo alternativas didáticas a serem trabalhadas em sala de aula.
\end{abstract}

Palavras-Chave: simuladores, aplicativo, ensino de Física, smartphone.

\footnotetext{
${ }^{1}$ Licenciatura em Física, Instituto Federal do Piauí-Campus Picos, marcosnd.31@gmail.com

${ }^{2}$ Licenciatura em Física, Instituto Federal do Piauí-Campus Picos, antonioedenilton10@outlook.com

${ }^{3}$ Licenciatura em Física, Instituto Federal do Piauí-Campus Picos, jailson_sva@hotmail.com

${ }^{4}$ Licenciatura em Física, Instituto Federal do Piauí-Campus Picos, rochaisaiane@gmail.com

5 Doutor em em Ciência e Engenharia de Materiais, Instituto Federal do Piauí-Campus Picos, haroldoram@ifpi.edu.br
} 


\begin{abstract}
With the constant advent of the evolution of Digital Information and Communication Technologies, there is a need for the school community to absorb and use these advances in order to promote a didactic and pedagogical renewal, in addition to following the Educational Laws, such as the Law of Guidelines and Bases of National Education (LDB) that cites the promotion of technological and scientific knowledge. In physics teaching, experimentation is of fundamental importance since it explicitly explains the concepts and theories through practice, and dynamizes the classes by providing the scientific vision of the area, surpassing the traditional methodologies. This article aims to propose a didactic alternative for the teaching of Physics through simulations of experiments with smartphones. The Physics Lab application, the subject of the study, was characterized within its potential use as a didactic tool for providing simulations of physical experiments in an intuitive and realistic manner. Through the Android platform, a pedagogical vision of its use as an auxiliary in the teaching of Electromagnetism and Electric Circuits was obtained, highlighting strengths and weaknesses within categories that can guide its use in the classroom. From this study, it was verified that the simulations provided by the application are valuable and can complement the theoretical approach overcoming a presumed lack of physical structure of specific laboratories. The preparation and orientation of the teacher is of fundamental importance for the success of the proposal, as it acts as a bridge between the students and the theoreticalpractical knowledge. In addition, it was exemplify possible methods of using the application by offering didactic alternatives to be worked in the classroom.
\end{abstract}

Keywords: simulators, app, physics teaching, smartphone.

\title{
Introdução
}

O ensino busca constante renovação das práticas pedagógicas, principalmente com o advento da era tecnológica, o que torna cada vez mais árdua a missão docente se levada em consideração as possíveis limitações encontradas em seu ambiente de trabalho, como a falta de subsídios físicos e materiais que promovam a inovação da didática.

As Tecnologias Digitais de Informação e Comunicação - TDIC's, são alvos constantes de estudos que abordam sua usabilidade no âmbito educacional. Diversos são os instrumentos da tecnologia passíveis de uso em sala de aula como ferramenta de ensino. Este artigo indica o smartphone, com aplicativos adequados, como potencial objeto de aliança entre o conhecimento teórico e prático, através de simulações. A possibilidade de realizar simulações dentro do ambiente virtual aliada a aceitável acessibilidade dos alunos a smartphones, podem validar a proposta dentro da Lei de Diretrizes e Bases da Educação Nacional (LDB) no tocante ao conhecimento científico-tecnológico.

Para atingir o objetivo do trabalho, foi utilizado o aplicativo Physics Lab disponível para plataforma Android (escolhida por ser a mais usual entre os alunos). O aplicativo foi caraterizado de acordo com suas potencialidades e limitações dentro do possível uso escolar 
como auxiliar no ensino do Eletromagnetismo e dos Circuitos Elétricos, funcionando como laboratório virtual. Assim, o trabalho busca tanto oferecer uma alternativa metodológica no ensino de Física, quanto propor a inclusão dos avanços tecnológicos no ambiente escolar, de forma a promover aprendizagem significativa.

\section{Fundamentação Teórica}

A era digital traz constantes mudanças à sociedade, desde a alta velocidade de comunicação à resolução de tarefas manuais mais complexas. A renovação do público alvo das escolas é acompanhada pela demanda de novas metodologias de ensino que se utilizem destes instrumentos tecnológicos. Neste cenário, as TDIC's devem atuar como ponte entre o conhecimento curricular e o conhecimento vivenciado (BRASIL, 1996).

A metodologia tradicional de ensino de Física ainda é prática comum nas salas de aula, tornando-as cansativas e matematizadas a ponto de afastarem os alunos e prejudicar seu desempenho. A experimentação no ensino é de fundamental importância pois revela o caráter científico e metodológico da disciplina e colabora para uma maior facilidade de compreensão por parte do aluno (FEIX, 2012).

Apesar de existir vários experimentos acessíveis para ensinar eletromagnetismo, o aluno continua não conseguindo visualizar alguns fenômenos, como as cargas elétricas, os polos magnéticos gerando linhas de campo, ou a relação entre eles. Logo, se vê uma dificuldade em abordar esse tópico somente em aulas expositivas tradicionais. Uma alternativa pode ser, portanto, o uso de simulações através das TDIC's, conforme relata Veras (2018, p. 24)

\footnotetext{
Acreditamos que as simulações computacionais são recursos pedagógicos valiosos que facilitam a aprendizagem e podem auxiliar a desmistificar a imagem da física no âmbito escolar, como uma disciplina difícil, que contém apenas fórmulas, um ramo da Matemática. Outro ponto que devemos salientar é familiaridade e o interesse desta geração atual de estudantes com aplicativos e atividades computacionais que geralmente fazem parte do cotidiano de muitos. (SOUZA, 2015, p. 19)
}

Como o smartphone é um dispositivo de fácil acesso a grande parte do público escolar, figura como um potencial instrumento de ensino (FONSECA, 2013). Com a grande presença destes dispositivos entre a comunidade, pode ser adotado como aliança entre a teoria 
e a prática, dinamizando as aulas e complementando o conhecimento dos alunos (SOUZA, 2017).

Cabe ao professor selecionar o que melhor atende à sua necessidade, assim como a de seus alunos, de forma que possa ajudá-los a compreender e ter uma melhor visão da disciplina. O apoio do professor é fundamental dentro do relacionamento entre TDIC's e alunos, já que grande parte sente dificuldade em utilizar as novas tecnologias com o intuito acadêmico, pois sua maior habilidade é utilizá-las dentro dos seus momentos de lazer. (FERNANDES, 2018)

O aplicativo Physics Lab desenvolvido pela empresa Fuzhou CIVITAS, é disponibilizado gratuitamente nas plataformas de dispositivos móveis Android e IOS. Seu uso requer um aparelho com sistema versão 8 ou acima para dispositivos IOS, e sistema versão 4.3 ou acima para dispositivos Android.

Dispõe de funcionamento baseado em redes sociais, onde o usuário pode adicionar amigos e interagir dentro de fóruns. Além disso para estimular o uso frequente, o aplicativo possui uma dinâmica de níveis, na qual o usuário aumenta seu nível a cada tarefa cumprida.

A aplicação conta com simulações da Física nas áreas da Astrofísica, Circuitos Elétricos e Eletromagnetismo.

\section{Metodologia}

A revisão do aplicativo Physics Lab foi realizada qualitativamente, na plataforma de dispositivos móveis Android, organizada dentro de categorias, que podem auxiliar quanto à aplicação em cada situação apontando vantagens e possíveis limitações em seu uso. As categorias consideradas foram:

- Interface: na qual foram destacados os principais pontos acerca dos componentes gráficos do aplicativo, quanto a facilidade de identificação dos itens e amigabilidade junto aos usuários.

- Linguagem: dentro dessa categoria está presente o que cabe à comunicação junto ao usuário. Os textos expostos nos menus e opções, línguas utilizadas e facilidade de compreensão em cada tópico foram algumas das relevâncias da categoria.

- Usabilidade: se refere à praticidade de uso geral, principalmente das simulações. O quão fácil é utilizar o aplicativo. 
- Fidelidade à teoria: como instrumento de apoio didático, as simulações do aplicativo devem ser fieis à teoria presente na literatura, de acordo com o abordado em sala de aula e principalmente, retratar precisamente os fenômenos reais. Neste item, avaliouse a mecânica do aplicativo, se corresponde de maneira satisfatória à realidade e a abordagem da disciplina de Física, no tocante ao Eletromagnetismo e Circuitos Elétricos presentes no currículo do ensino médio.

As categorias foram organizadas de forma que explicitassem principalmente as características pedagógicas, quanto sua aplicação e uso voltado para o ensino. Por delimitação de estudo, somente as simulações de Circuitos Elétricos e Eletromagnetismo foram consideradas na análise.

\section{Resultados e Discussão}

Tabela 1 - Análise do aplicativo Physics Lab por categoria. Fonte: Própria

\begin{tabular}{|l|l|}
\hline INTERFACE & $\begin{array}{l}\text { O layout das telas é bem simples, a primeira contém em } \\
\text { destaque as opções de simulação disponíveis. Outros }\end{array}$ \\
& itens são bem caracterizados através de imagens que \\
& descrevem suas funções, como configurações, \\
& notificações, amizades, tela inicial, experimentos e \\
& fórum, que são os principais; \\
- & $\begin{array}{l}\text { Todas as animações são coloridas e bem texturizadas, } \\
\text { procurando explicitar o aspecto realista de cada um dos } \\
\text { objetos dentro das simulações; }\end{array}$ \\
& $\begin{array}{l}\text { As simulações foram desenvolvidas em interface 3D, } \\
\text { dando profundidade às imagens. }\end{array}$ \\
\hline LINGUAGEM & $\begin{array}{l}\text { Um ponto negativo a ser levantado é a não tradução do } \\
\text { aplicativo para a língua portuguesa, o que pode ser um } \\
\text { obstáculo. As línguas presentes são: mandarim, inglês, } \\
\text { francês, alemão e japonês; } \\
\text { Apesar disto, o uso pode ser intuitivo pela presença de } \\
\text { imagens que traduzem a função de cada opção, guiando o } \\
\text { usuário a opção desejada. }\end{array}$ \\
\hline
\end{tabular}




\begin{tabular}{|c|c|}
\hline USABILIDADE & $\begin{array}{l}\text { - O aplicativo se mostra bem fluido dependendo do } \\
\text { desempenho técnico do smartphone. Como é uma } \\
\text { aplicação bem leve, pode ser utilizada nos aparelhos } \\
\text { básicos recentes; } \\
\text { - O aplicativo não precisa estar conectado à internet, } \\
\text { podendo ser trabalhado em qualquer ambiente, dentro ou } \\
\text { fora da sala de aula; } \\
\text { - Dentro das simulações, tem a mecânica de "clique e } \\
\text { arraste", dando dinamização à montagem dos } \\
\text { experimentos; } \\
\text { - O professor como mediador em sala de aula entre o } \\
\text { aplicativo e o aluno, pode auxiliar o seu uso, desde que } \\
\text { possua conhecimento básico na manipulação de } \\
\text { aplicações móveis. }\end{array}$ \\
\hline FIDELIDADE Å TEORIA & $\begin{array}{l}\text { - O aplicativo deve ser trabalhado como complemento às } \\
\text { aulas teóricas, já que oferece uma boa aproximação à } \\
\text { realidade - principalmente nos componentes dos circuitos } \\
\text { elétricos - e à teoria presente no currículo escolar; } \\
\text { - Nas simulações pode se encontrar diversos instrumentos } \\
\text { e dados físicos pertinentes, oferecendo alternativas de } \\
\text { trabalho didático ao professor. } \\
\text { - A realidade dos equipamentos é demonstrada também } \\
\text { pelos possíveis problemas que o usuário encontraria se } \\
\text { montasse o experimento real de maneira errada. Um } \\
\text { exemplo é a queima da bateria se ligada em curto } \\
\text { circuito, sendo necessária sua troca. } \\
\text { O aplicativo dispõe de experimentos pré-configurados, } \\
\text { facilitando o uso e agilizando o trabalho em sala. } \\
\text { Também é possível salvar experimentos, podendo ser } \\
\text { reutilizados em outro momento. }\end{array}$ \\
\hline
\end{tabular}

De modo geral, o aplicativo possui potencial por apresentar design simplista, limpo de poluições visuais, que contorna a falta de tradução com a clareza na apresentação das imagens nos itens.

Figura 1 - Tela inicial do aplicativo Physics Lab. Fonte: Própria. 


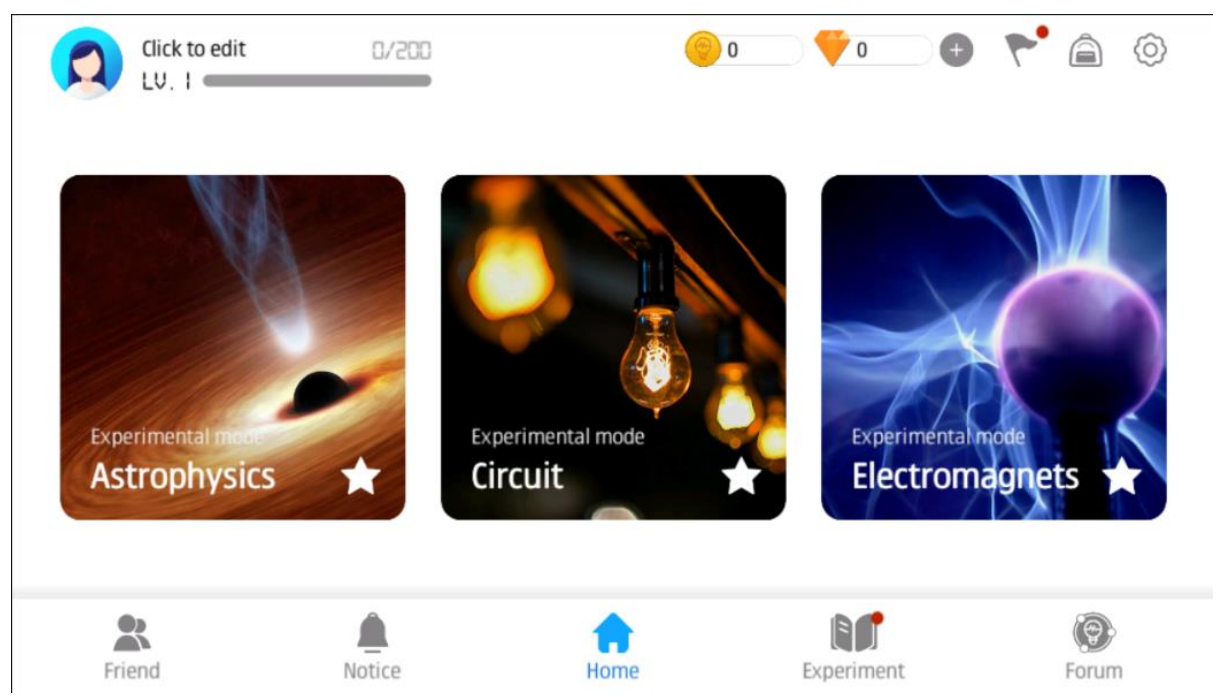

A tela de experimentos de Circuitos Elétricos é bastante realista, transparecendo fidelidade e auxiliando na montagem dos circuitos. A dinâmica é interativa, e com itens simples é possível reproduzir diversas configurações de circuitos. Há de se destacar também a variedade de componentes que o aplicativo oferece, ampliando as alternativas de montagens.

Figura 2 - Experimento de Circuito Elétrico feito no aplicativo Physics Lab. Fonte:

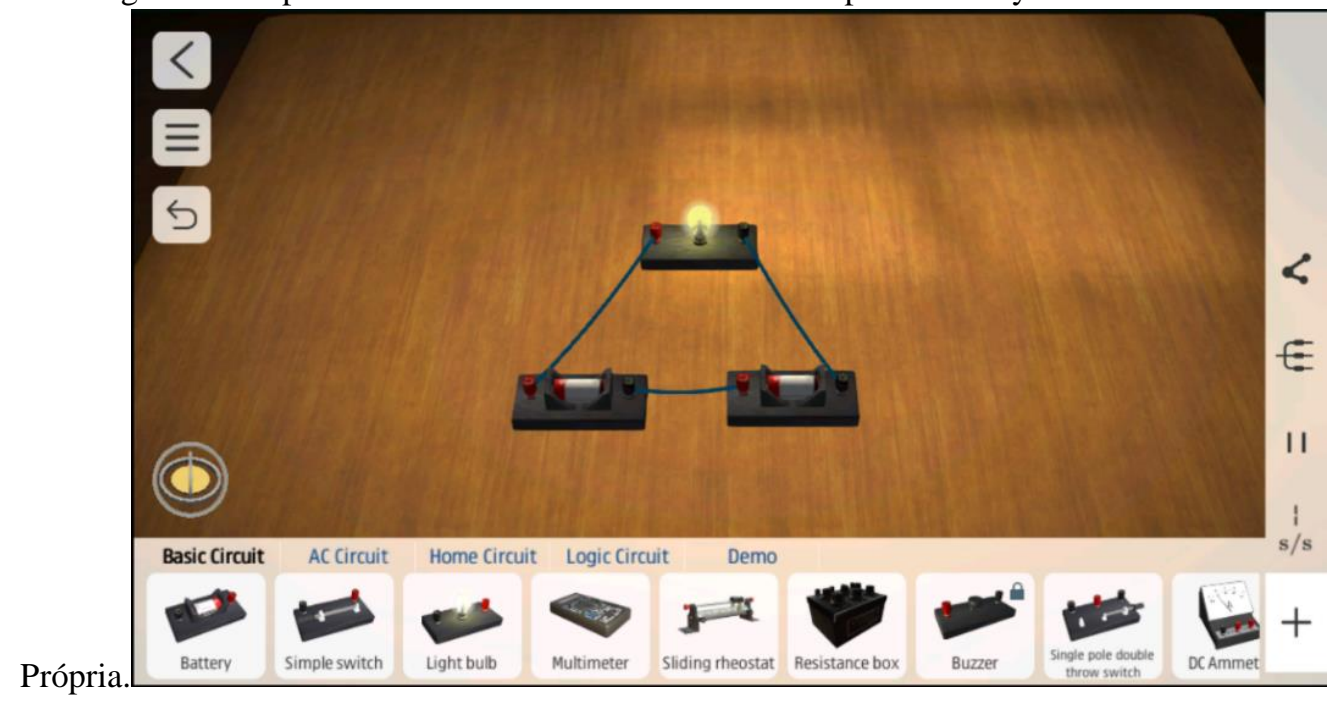

Os experimentos em Eletromagnetismo fornecem uma visão ideal das interações entre cargas e campos, elétricos e magnéticos. Dessa forma a compreensão pode se tornar mais visual e concreta.

Figura 3 - Experimento de Eletrostática feito no aplicativo Physics Lab. Fonte Própria. 


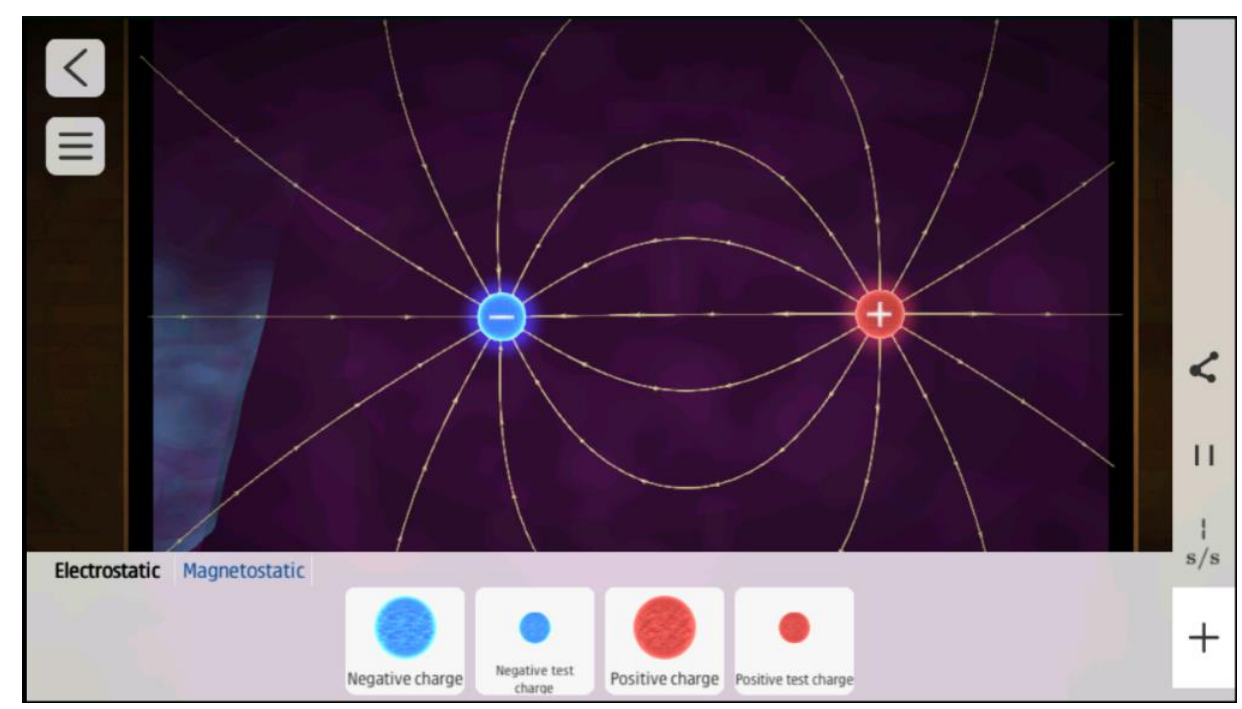

\section{Conclusões}

O aplicativo Physics Lab foi analisado com o intuito de demonstrar sua capacidade de complementação prática dentro da experimentação no ensino de Física. A maior desvantagem encontrada foi a não tradução da aplicação para a língua portuguesa. Apesar disso, oferece alternativas dentro da interface que podem orientar seu uso individual ou coletivo. Nessa perspectiva, o professor tem papel fundamental na ponte entre o simulador e aluno de forma a produzir conhecimento em conjunto.

O uso dos smartphones dentro do ensino de Física pode superar a falta de materiais e infraestrutura, servindo como alternativa à experimentação a partir de simulações, além de contribuir para o letramento tecnológico voltado para o ensino.

Possibilidades de uso do aplicativo dentro do estudo do Eletromagnetismo e Circuitos Elétricos em sala de aula são:

- Trabalhar em grupo o conceito dos elementos do circuito elétrico, propondo desafios de montagem de circuitos;

- Trabalhar individualmente a visualização dos campos elétrico e magnético, assim como a interação entre cargas e imãs;

- Demonstrar experimentos de laboratórios com as simulações oferecidas;

- Trabalhar com atividades extraclasse relacionadas ao conteúdo dentro das simulações.

\section{Referências}


BRASIL. LEI No 9.394, DE 20 DE DEZEMBRO DE 1996. Lei de Diretrizes e Bases da Educação Nacional, Brasília, DF, dez. 1996. Disponível em: <http://www.planalto.gov.br/CCIVIL_03/Leis/L9394.htm >. Acesso em: 19 set. 2018.

FEIX, E. C.; SARAIVA, S.B.; KIPPER, L. M. A importância da Física experimental no processo ensino-aprendizagem. III Salão de Ensino e de Extensão. ISSN 22379193. Santa Cruz do Sul, RS. 2012.

FERNANDES, Renato Izac et al. Metodologias ativas e Tecnologias na educação no ensino de Física. Anais do Simpósio Ibero-Americano de Tecnologias Educacionais, [S.1.], p. 15-21, junho 2018. ISSN 2594-388X. Disponível em: <https://publicacoes.rexlab.ufsc.br/index.php/sited/article/view/153>. Acesso em: 19 set. 2018.

FONSECA, Ana Graciela Mendes Fernandes da. APRENDIZAGEM, MOBILIDADE E CONVERGÊNCIA: Mobile Learning com Celulares e Smartphones. Revista mídia e cotidiano, [s.1.], v. 2, n. 2, p.265-283, 30 jun. 2013. Pro Reitoria de Pesquisa, Pós-Graduação e Inovação - UFF. http://dx.doi.org/10.22409/ppgmc.v2i2.9685.

SOUZA, Gláucia Martins Ricardo et al. Uso de simulações computacionais no ensino de conceitos de força e movimento no $9^{\circ}$ ano do Ensino Fundamental. 2015. 192p. Dissertação (Mestrado Profissional do Ensino de Física (MNPEF)). Universidade Federal Fluminense, 2015. em: https://app.uff.br/riuff/bitstream/1/4227/1/G1\%C3\%A1ucia\%20Martins\%20Ricardo \%20Souza\%20-\%20Disserta\%C3\%A7\%C3\%A3o\%20Final.pdf. Acesso em 19 set. 2018.

SOUZA, Paulo Henrique da Silveira et al. Aplicativos educacionais: um estudo de caso no desenvolvimento de um aplicativo na plataforma App Inventor2 para auxílio no ensino de produção textual nas aulas de português. 2017.

VERAS, Ricardo Silva. Simulações digitais com uso do PhET para o ensino e aprendizagem de força e movimento. 2018. 76 p. Trabalho de Conclusão de Curso de Graduação (Licenciatura em Física) - Universidade do Estado do Amazonas, Manaus, 2018. Disponível em: <http://repositorioinstitucional.uea.edu.br/handle/riuea/984>. Acesso em: 19 set. 2018. 